BU-HEPP-02/02

CASPER-02/02

hep-ph/0209050

August 2002

\title{
On the Possibility of Optical Unification in Heterotic Strings
}

\author{
G. Cleaver, ${ }^{1,2 *}$ V. Desai, ${ }^{1,3 \dagger}$ H. Hanson, ${ }^{1,4 \ddagger}$ J. Perkins, ${ }^{1 \S}$ \\ D. Robbins, ${ }^{1,5} \&$ S. Shields ${ }^{1,6 \|}$ \\ ${ }^{1}$ Center for Astrophysics, Space Physics \& Engineering Research, \\ Dept. of Physics, PO Box 97316, Baylor University, \\ Waco, TX 76798-7316, USA \\ 2 Astro Particle Physics Group, Houston Advanced Research Center (HARC), \\ The Mitchell Campus, Woodlands, TX 77381, USA \\ ${ }^{3}$ St. Thomas Episcopal School, \\ 4900 Jackwood, Houston, TX 77096, USA, \\ ${ }^{4}$ Department of Physics, University of West Florida, \\ Pensacola, FL 32514, USA \\ ${ }^{5}$ Department of Physics, Tulane University, \\ New Orleans, LA 70118, USA \\ ${ }^{6}$ Department of Physics, California Polytechnic State University, \\ San Luis Obispo, CA 9340\%, USA
}

*Gerald_Cleaver@baylor.edu

†verlox7516@yahoo.com

$\ddagger^{\ddagger}$ hah1@students.uwf.edu

§John_Perkins@baylor.edu

ฯdrobbin@tulane.edu

" sshields@calpoly.edu 


\begin{abstract}
Recently J. Giedt discussed a mechanism, entitled optical unification, whereby string scale unification is facilitated via exotic matter with intermediate scale mass. This mechanism guarantees that a virtual MSSM unification below the string scale is extrapolated from the running of gauge couplings upward from $M_{Z^{\circ}}$ when an intermediate scale desert is assumed. In this letter we explore the possibility of optical unification within the context of weakly coupled heterotic strings. In particular, we investigate this for models of free fermionic construction containing the NAHE set of basis vectors. This class is of particular interest for optical unification, because it provides a standard hypercharge embedding within $S O(10)$, giving the standard $k_{Y}=\frac{5}{3}$ hypercharge level, which was shown necessary for optical unification. We present a NAHE model for which the set of exotic $S U(3)_{C}$ triplet/anti-triplet pairs, $S U(2)_{L}$ doublets, and non-Abelian singlets with hypercharge offers the possibility of optical unification. Whether this model can realize optical unification is conditional upon these exotics not receiving Fayet-Iliopoulos (FI) scale masses when a flat direction of scalar vacuum expectation values is non-perturbatively chosen to cancel the FI $D$-term $\xi$ generated by the anomalous $U(1)$-breaking Green-Schwarz-Dine-Seiberg-Wittten mechanism. A study of perturbative flat directions and their phenomenological implications for this model is underway.

This paper is a product of the NFS Research Experiences for Undergraduates and the NSF High School Summer Science Research programs at Baylor University.
\end{abstract}




\section{Optical Unification}

An enduring issue in string theory has been the discrepancy between the $S U(3)_{C} \times S U(2)_{L} \times U(1)_{Y}([321])$ gauge coupling unification scale $\Lambda_{U}$ for the Minimal Supersymmetric Standard Model (MSSM) with intermediate scale desert and the string scale $\Lambda_{H}$ for the weakly coupled heterotic string. When couplings are run upward from their values near $M_{Z^{o}}$, a MSSM unification scale $\Lambda_{U} \sim 2.5 \times 10^{16} \mathrm{GeV}$ [1] is predicted for an intermediate scale desert. In contrast, the weakly coupled heterotic string scale is around $\Lambda_{H} \sim 5 \times 10^{17}$ [2].

Three types of solutions have been proposed to resolve this factor of 20 disagreement [3]. One proposal is a grand unified theory between $\Lambda_{U}$ and $\Lambda_{H}$. Here the MSSM couplings merge at $\Lambda_{U}$ and then run together within a GUT to $\Lambda_{H}$. However, with the exception of flipped $S U(5)$ [4] (or partial GUTs such as the Pati-Salam $\left.S U(4)_{C} \times S U(2)_{L} \times S U(2)_{R}[5]\right)$ string GUT models based on level-one Kač-Moody algebras encounter a difficulty: they lack the required adjoint higgs (and higher representations). Alternately, strong coupling effects of $M$-theory can lower $\Lambda_{H}$ down to $\Lambda_{U}$ [6]. Conversely, intermediate scale exotics could shift the MSSM unification scale upward to the string scale [7].

The near ubiquitous appearance of MSSM-charged exotics in heterotic string models adds weight to the third proposal.* If MSSM exotics exist with intermediate scale masses of order $\Lambda_{I}$, then the actual [321] running couplings are altered above $\Lambda_{I}$. It is then, perhaps, phenomenologically puzzling that the illusion of MSSM unification should still be maintained when the intermediate scale MSSM exotics are ignored [11]. Maintaining this illusion likely requires very fine tuning of $\Lambda_{I}$ for a generic exotic particle set and $\Lambda_{H}$. Slight shifting of $\Lambda_{I}$ would, with high probability, destroy appearances. Thus, in some sense, the apparent MSSM unification below the string scale might be viewed as accidental $[12,11]$.

A mechanism whereby the appearance of a $\Lambda_{U}$ is not accidental would be very appealing. Just such a mechanism, entitled "optical unification," has recently been discussed by Joel Giedt [11]. Optical unification results in $\Lambda_{U}$ not disappearing under shifts of $\Lambda_{I}$. Instead, $\Lambda_{U}$ likewise shifts in value. This effect is parallel to a virtual image always appearing between a diverging lens and a real object, independent of the position of the lens or real object. Hence, Giedt's choice of appellation for this mechanism.

Successful optical unification requires three things [11]. First, the effective level of the hypercharge generator must be the standard

$$
k_{Y}=\frac{5}{3} .
$$

*Even the underlying model from which the Minimal Supersymmetric Heterotic String Model [MSHSM] is derived contains MSSM-charged exotics [8]. These exotics are, however, eliminated from the MSHSM low energy effective field theory by a set of anomaly-cancelling flat directions $[9,10]$. 
(1.1) is a strong constraint on string-derived [321] models, for the vast majority have non-standard hypercharge levels [13]. Only select classes of models, such as the NAHE-based [14] free fermionic class [15], can yield $k_{Y}=\frac{5}{3} \cdot{ }^{\dagger}$ Second, optical unification imposes the relationship

$$
\delta b_{2}=\frac{7}{12} \delta b_{3}+\frac{1}{4} \delta b_{Y} .
$$

between the exotic particle contributions $\delta b_{3}, \delta b_{2}$, and $\delta b_{1}$ to the [321] beta function coefficients. Each $S U(3)_{C}$ exotic triplet or anti-triplet contributes $\frac{1}{2}$ to $\delta b_{3}$; each $S U(2)_{C}$ doublet contributes $\frac{1}{2}$ to $\delta b_{2}$. With the hypercharge of a MSSM quark doublet normalized to $\frac{1}{6}$, the contribution to $\delta b_{Y}$ from an individual particle with hypercharge $Q_{Y}$ is $Q_{Y}^{2} . \delta b_{3}>\delta b_{2}$ is required to keep the virtual unification scale below the string scale. Combining this with (1.2) imposes

$$
\delta b_{3}>\delta b_{2} \geq \frac{7}{12} \delta b_{3}
$$

since $\delta b_{Y} \geq 0$.

To acquire intermediate scale mass, the exotic triplets and anti-triplets must be equal in number. Similarly, the exotic doublets must be even in number. Hence, $\delta b_{3}$ and $\delta b_{2}$ must be integer [11]. As Giedt pointed out, the simplest solution to (1.2) and (1.3) is a set of three exotic triplet/anti-triplet pairs and two pairs of doublets. One pair of doublets can carry $Q_{Y}= \pm \frac{1}{2}$, while the remaining exotics carry no hypercharge. Alternately, if the doublets carry too little hypercharge, some exotic $S U(3)_{C} \times S U(2)_{L}$ singlets could make up the hypercharge deficit. The next simplest solution requires four triplet/anti-triplet pairs and three pairs of doublets that yield $\delta b_{Y}=2 \frac{2}{3}$ either as a set, or with the assistance of additional non-Abelian singlets. For more than four $3 / \overline{3}$ pairs, (1.2) and (1.3) allow more than one number of doublet pairs.

\section{Possible Optical Unification in Heterotic Strings}

An excellent class of string models to investigate for possible optical unification is heterotic models of free fermionic construction containing the NAHE set. In particular, these models can always provide a standard $k_{Y}=\frac{5}{3}$ hypercharge embedding $[4,16,17,18] .(1.1)$ is a very non-trivial constraint which generally cannot be satisfied for models of other classes [13]. Thus, we re-examined the known NAHE-based [321] models [16, 17, 18, 19, 20, 21] and [322] left-right symmetric extensions [22, 23] with regard to their MSSM exotic particle content.

Generic NAHE-based [321] models contain a only few exotic $S U(3)_{C}$ triplet/antitriplet pairs. The models of [16], [17], and [18] contain one pair, two pairs, and one

†The "NAHE" set is named after Nanopoulos, Antoniadis, Hagelin and Ellis and refers to a specific set of of five free fermionic basis vectors, which first appeared in the construction of stringbased flipped $S U(5)$. 
pair, respectively The models of [19], [20], and Table 5 model of [21] each contain 3 pairs. The [322] left-right symmetric models 1, 2, and 3 of [22] contain one, four, and two pairs, while the model of [23] contains three pairs. Thus, the models of [16], [17], and [18], along with models 1 and 3 of [22], contain too few $S U(3)$ exotics to generate optical unification.

The basis vectors of the [18], [19], and [20] models are the same. The variations among the corresponding models are solely a function of some differing GSO phase choices. Noting this, we have investigated further variations in GSO phase choices for these basis vectors. ${ }^{\ddagger}$ Our investigation has resulted in five additional models, denoted FCREU1 through FCREU5, which respectively contain four pairs, three pairs, two pairs, no pairs, and, again, no pairs of exotic triplets/anti-triplets. The gauge groups and MSSM-charged exotic particle content of these models appear in Table A1. The corresponding GSO phase variations from those of [18] are listed in Table A2.

Of the [321] models with three or more exotic $3 / \overline{3}$ pairs, we focus on model FCREU1 with its 4 exotic pairs. (In Table A3 we list the complete set of states of this model and their charges.) Each of the other models presents difficulties: The model of [20] contains too few exotic doublets (only three rather than four) to satisfy (1.3). The model FCREU2 with three exotic triplet/anti-triplet pairs contains nine exotic $S U(2)_{L}$ doublets, but six of these doublets are coupled in pairs via a custodial $S U(2)_{C}$. Further, with regard to MSSM exotics, model FCREU1 is an enhanced version of the model in [19]. Any optical solution involving exactly three triplet/antitriplet pairs from model FCREU1 is also a solution for the model of [19] (and vice versa). The left-right symmetric models of [22] and [23] with three or more exotic $3 / \overline{3}$ pairs will be discussed in a separate paper.

The MSSM-charged exotics of model FCREU1 are four $S U(3)_{C} 3 / \overline{3}$ pairs, three $S U(2)_{L}$ doublet pairs, and seven pairs of MSSM exotic non-Abelian singlets carrying $Q_{Y}= \pm \frac{1}{2}$, in addition to three pairs of linear combinations of the MSSM higgs-like doublets, $h_{i=1 \text { to } 4}$ and $\bar{h}_{i=1 \text { to } 4}$. The extra higgs-like doublets are generally present in NAHE-based [321] models. Three of the $S U(3)_{C}$ exotic pairs carry $Q_{Y}= \pm \frac{1}{3}$, while the remaining pair carries $Q_{Y}= \pm \frac{1}{6}$. None of the exotic $S U(2)_{L}$ non-higgs doublets carry hypercharge. For successful optical unification, when all four $3 / \overline{3}$ exotic pairs receive intermediate scale masses, all three pairs of exotic non-higgs doublets must also. Further, the total contribution to $\delta b_{Y}$ from all $3 / \overline{3}$ pairs is $2 \frac{1}{6}$, while $(1.2)$ requires a total $\delta b_{Y}=2 \frac{2}{3}$. Since the exotic non-higgs doublets have no hypercharge, the remainder of $2 \frac{2}{3}-2 \frac{1}{6}=\frac{1}{2}$ must be provided by exactly two exotic singlets, each contributing $\frac{1}{4}$ to $\delta b_{Y}$. The six remaining pairs of exotic singlets must acquire FayetIliopoulos (FI) scale masses.

If one exotic $3 / \overline{3}$ pair receives near string scale mass, leaving only three exotic $3 / \overline{3}$ pairs to receive intermediate scale masses, the total triplet/anti-triplet contribution

\footnotetext{
${ }^{\ddagger}$ Because of the symmetries within the basis vectors generating these models, differing GSO phase choices can yield identical models. The complete set of models produced from the distinct GSO phase choices for the basis vectors of [18] will be discussed elsewhere.
} 
to $\delta b_{Y}$ can be $2,1 \frac{3}{4}$, or $1 \frac{1}{2}$, depending on whether zero, one, or two intermediate scale (anti-)triplets carry $Q_{Y}= \pm \frac{1}{6}$, respectively. Even the lowest of these choices provides too large of a contribution to $\delta b_{Y}$, since for three exotic $3 / \overline{3}$ pairs (1.2) and (1.3) require $\delta b_{Y}=1$. Hence, optical unification cannot be achieved for this model when only three $3 / \overline{3}$ pairs acquire intermediate scale masses. This also implies optical unification is not possible for the model of [19]. The Table 5 model of [21] is another with three exotic $3 / \overline{3}$ pairs, of which each has $Q_{Y}= \pm \frac{1}{3}$. Thus, the Table 5 model is similarly prohibited from optical unification.

Optical lensing might be possible in other models containing exactly three hypercharged exotic $3 / \overline{3}$ pairs, if these pairs were of the $Q_{Y}= \pm \frac{1}{6}$ class. Then their total contribution to $\delta b_{Y}$ would be $\frac{1}{2}$. The remaining contribution of $\frac{1}{2}$ for the required total $\delta b_{Y}=1$ could be provided by two singlets, each with $Q_{Y}= \pm \frac{1}{2}$. A 3 or $\overline{3}$ with $Q_{Y}= \pm \frac{1}{3}$ originates in a sector for which the fermions generating the $S U(3)_{C}$ symmetry have antiperiodic boundary conditions, while a 3 or $\overline{3}$ with $Q_{Y}= \pm \frac{1}{6}$ originates in a sector for which these fermions have periodic boundary conditions. Whether or not a model can have exactly three exotic $3 / \overline{3}$ pairs with $Q_{Y}= \pm \frac{1}{6}$ is under investigation $[24]$.

In addition to containing the set of four $3 / \overline{3}$ pairs, three non-higgs-like doublet pairs, and one pair of singlets, which satisfies the optical unification constraints (1.1)(1.3), model FCREU1 possesses the other six pairs of $Q_{Y}= \pm \frac{1}{2}$ singlets and the three extra higgs-like doublets. For successful optical unification these additional MSSM exotics must receive near string scale masses, while the optical unifying set does not.

Like the other NAHE-based standard-like models, the model of [18], and its variations in $[19,20,21]$ and herein, contain an anomalous $U(1)[25] . \S$ The anomalous Abelian symmetry is broken by the Green-Schwarz-Dine-Seiberg-Witten mechanism [26], which generates a contribution, $\xi$, to the anomalous FI $D$-term in the process. A flat direction of vacuum expectation values (VEVs) of scalars is then non-perturbatively chosen to cancel the FI $\xi$-term, restoring supersymmetry and stabilizing the vacuum. The FI VEV scale is typically $\sim \frac{1}{10}$ of the string scale. Superpotential interaction of the flat direction VEVs with superfields can generate (near) FI scale masses for various states. Thus, ideally it may be possible for the six additional pairs of exotic singlets and three pairs of extra higgs-like doublets to receive FI scale masses from the flat direction VEVs, while the optical unification exotics remain massless at the FI scale.

Singlet and non-singlet flat directions of the MSHSM were constructed in $[8,9,10]$ that give FI scale masses to exactly three out of four pairs of higgs-like doublets. For these flat directions, the physical higgs doublets $h$ and $\bar{h}$ are formed from a linear combination of three or more $h_{i}$ and $\bar{h}_{i}$, respectively, while three orthogonal linear combinations of $h_{i}$ and of $\bar{h}_{i}$ receive FI scale mass. Similar flat directions may accomplish this for model FCREU1 also. Such flat directions must also generate

${ }^{\S}$ A few semi-realistic non-anomalous NAHE-based models have been constructed, such as the first two left-right symmetric models in [22], but these have enhanced observable sector symmetries. 
FI scale masses for exactly six of the seven pairs of exotic $Q_{Y}$-carrying singlets. Interestingly, as Table $\mathrm{A} 3$ indicates, the pair of singlets denoted $A_{1} / \bar{A}_{1}$ does not follow the charge pattern of the other six. This is the singlet pair most likely to remain massless at the FI scale. To be consistent with optical unification, a flat direction must, of course, keep the four pairs of exotic MSSM triplets and the three pairs of exotic non-higgs-like doublets FI scale massless, as we have discussed. Studies of model FCREU1 perturbative flat directions and of their implications regarding masses are underway [24]. The methods of this investigation are parallel to those followed in the flat direction studies of the MSHSM [8, 9, 10], flipped SU(5) [27, 28], and of other standard-like and semi-GUT models [22, 23, 29, 30] located in the parameter space of NAHE-based models.

Assuming that the required exotic triplets, doublets, and non-Abelian singlets remain massless at the FI scale, an intermediate mass scale must be generated for them, perhaps through ninth or higher order mass terms resulting from flat direction VEVs or hidden sector condensation. Mass terms for specific flat directions will be studied in [24]. Alternately, for a generic $S U\left(N_{c}\right)$ gauge group containing $N_{f}$ flavors of matter states in vector-like pairings $H_{i} \bar{H}_{i}, i=1, \ldots N_{f}$, the gauge coupling $g_{i}$, though weak at the string scale $M_{s t r}$, becomes strong for $N_{f}<3 N_{c}$ at a condensation scale defined by

$$
\Lambda=M_{P L} \mathrm{e}^{8 \pi^{2} / \beta g_{s}^{2}},
$$

where the $\beta$-function is given by,

$$
\beta=-3 N_{c}+N_{f} .
$$

The $N_{f}$ flavors counted are only those that ultimately receive masses $m \ll \Lambda$. The hidden sector matter states of model FCREU1 are four $5 / \overline{5}$ pairs of $S U(5)_{H}$ and four $3 / \overline{3}$ pairs of $S U(3)_{H}$. Any number of these states from none to all might become FI scale massive under a flat direction. Thus, $\beta_{5}$ could be anywhere from -15 to -11 , corresponding to a $S U(5)$ condensate scale range of $1 \times 10^{14} \mathrm{GeV}$ to $2 \times 10^{15} \mathrm{GeV}$. Similarly, $\beta_{3}$ could be anywhere from -9 to -5 , corresponding to a $S U(3)$ condensate scale range of $1 \times 10^{9} \mathrm{GeV}$ to $2 \times 10^{13} \mathrm{GeV}$. Thus, when generated by hidden sector condensation, the intermediate mass scale for the MSSM exotics could be anywhere from $10^{9} \mathrm{GeV}$ to $10^{15} \mathrm{GeV}$.

\section{Concluding Comments}

In this letter we have discussed the possibility of achieving optical unification within a [321] heterotic string. Optical unification, recently suggested by J. Giedt [11], would explain the apparent MSSM unification scale near $2.5 \times 10^{16} \mathrm{GeV}$ as a "virtual image effect" of an actual unification of couplings at the string scale, $\sim 5 \times 10^{17} \mathrm{GeV}$. An intriguing aspect of optical unification is that the apparent MSSM 
unification is not accidental. Rather, in this case, like the guaranteed appearance of a virtual image between a diverging lens and a real object, a MSSM unification scale will always appear between the intermediate mass scale of the MSSM exotics and the string unification scale, when coupling strengths are run upward from their measured values at low energy scales, under the assumption of an intermediate scale desert. As movement of the diverging lens or of the real object simply alters the position of the virtual image, so too movement of the intermediate mass scale or of the string scale simply alters the location of the predicted MSSM unification scale. Relatedly, detection of an intermediate exotic MSSM mass scale in combination with the currently extrapolated MSSM unification scale would, thus, reveal the string scale.

We presented herein a model possessing the potential for optical unification. This model is of free fermionic construction in the NAHE class. Its basis vectors first appeared in $[18,19,20]$. The differences between this model and those in $[18,19,20]$ result from a few changes in GSO phases. These changes produce a set of MSSM exotic states with properties strongly suggesting that optical unification may be possible within some regions of the parameter space of NAHE-based weakly coupled heterotic strings. Further research will reveal if this is indeed so [24].

\section{Acknowledgments}

This letter is a product of the 2002 NSF High School Summer Science Research (HSSSR) and Research Experiences for Undergraduates (REU) programs sponsored by the Center for Astrophysics, Space Physics, and Engineering Research (CASPER) at Baylor University. Research funding for Viren Desai was provided by the HSSSR program; Research funding for Heather Hanson, David Robbins, and Scot Shields was provided by the REU program. G.C. thanks Alon Faraggi and Dimitri Nanopoulos for numerous helpful discussions regarding NAHE-based models and thanks Joel Giedt for helpful discussions regarding optical unification. 


\section{References}

[1] J. Ellis, S. Kelley and D.V. Nanopoulos, Phys. Lett. B249 (1990) 441;

U. Amaldi, W. de Boer and F. Fürstenau, Phys. Lett. B260 (1991) 447;

P. Langacker and M. Luo, Phys. Rev. D44 (1991) 817;

A.E. Faraggi and B. Grinstein, Nucl. Phys. B422 (1994) 3, [hep-th/9308329];

D.M. Ghilencea and G.G. Ross, Nucl. Phys. B606 (2001) 101, [hep-ph/0102306].

[2] V.S. Kaplunovsky, Nucl. Phys. B307 (1988) 145; Erratum Nucl. Phys. B382 (1992) 436.

[3] For a summary of the various proposed solutions see for example,

K.R. Dienes and A.E. Faraggi, Phys. Rev. Lett. 75 (1995) 2646, [hepth/9505018]; Nucl. Phys. B457 (1995) 409, [hep-th/9505046];

K.R. Dienes, Phys. Rep. 287 (1997) 447, [hep-th/9602045], and references therein.

[4] I. Antoniadis, J. Ellis, J. Hagelin, and D.V. Nanopoulos, Phys. Lett. B231 (1989) 65 ;

I. Antoniadis, J. Ellis, R. Lacaze, D.V. Nanopoulos, Phys. Lett. B268 (1991) 188 ;

J. Lopez, D.V. Nanopoulos and K. Yuan, Nucl. Phys. B399 (1993) 654;

J. Lopez and D.V. Nanopoulos, "Flipped SU(5): A Grand Unified Superstring Theory (GUST) Prototype," [hep-ph/9511266];

A. Dedes, C. Panagiotakopoulos, K. Tamvakis, Phys. Rev. D57 (1998) 5493, [hep-ph/9710563];

K.T. Mahanthappa, Phys. Lett. B441 (1998) 178, [hep-ph/9807231];

A.E. Faraggi, R. Garavuso, J.M. Isidro, "Nonperturbative Flipped SU(5) vacua in heterotic M Theory," OUTP-02-15P, [hep-ph/0204080].

[5] J. Pati and R.N. Mohapatra, Phys. Rev. D11 (1975) 2558;

D. Chang, R.N. Mohapatra, M.K. Parida, Phys. Rev. D30 (1984) 1052;

I. Antoniadis, G.K. Leontaris, and J. Rizos, Phys. Lett. B245 (1990) 161;

G.K. Leontaris, and J. Rizos, Nucl. Phys. B554 (1999) 3;

F. Astorga, AIP Conf.Proc. 478 (1999) 277;

Y. Mimura, S. Nandi, Phys. Lett. B538 (2002) 406, [hep-ph/0203126].

[6] E. Witten, Nucl. Phys. B471 (1996) 135, [hep-th/9602070].

[7] See for example,

S. Chang, C. Coriano, and A. Faraggi, Phys. Lett. B397 (1997) 76, [hep$\mathrm{ph} / 9603272]$. 
[8] G.B. Cleaver, A.E. Faraggi and D.V. Nanopoulos, Phys. Lett. B455 (1999) 135; G.B. Cleaver, "M-Fluences on String Model Building," CTP-TAMU-46/98, [hep$\mathrm{ph} / 9901203]$.

[9] G.B. Cleaver, A.E. Faraggi and D.V. Nanopoulos, Int. J. Mod. Phys. A16 (2001) 425, [hep-ph/9904301];

G.B. Cleaver, A.E. Faraggi, D.V. Nanopoulos, and J.W. Walker, Nucl. Phys. B593 (2001) 471, [hep-ph/9910230].

[10] G.B. Cleaver, A.E. Faraggi, D.V. Nanopoulos, and J.W. Walker, Mod. Phys. Lett. A15 (2000) 1191, [hep-ph/0002060]; Nucl. Phys. B620 (2002) 259, [hep$\mathrm{ph} / 0104091]$

G.B. Cleaver, "Phenomenological Survey of a Minimal Superstring Standard Model," [hep-ph/0003208]; Int. J. Mod. Phys. A16S1C (2001) 949, [hep$\mathrm{ph} / 0011020]$.

[11] J. Giedt, "Optical Unification," LBNL-50358, UCB-PTH-02/23, [hep$\mathrm{ph} / 0205224]$.

[12] D. Ghilencea, Phys. Lett. B456 (1999) 540, [hep-ph/9904293].

[13] J. Giedt, "Heterotic Orbifolds," Ph.D. Thesis, LBNL-50169, UCB-PTH-02/19, [hep-ph/0204315].

[14] A.E. Faraggi and D.V. Nanopoulos, Phys. Rev. D48 (1993) 3288;

A.E. Faraggi, [hep-th/9511093]; [hep-th/9708112].

[15] I. Antoniadis, C. Bachas, and C. Kounnas, Nucl. Phys. B289 (1987) 87;

H. Kawai, D.C. Lewellen, and S.H.-H. Tye, Nucl. Phys. B288 (1987) 1.

[16] A.E. Faraggi, D.V. Nanopoulos, and K. Yuan, Nucl. Phys. B335 (1990) 347;

A.E. Faraggi, Phys. Rev. D46 (1992) 3204.

[17] A.E. Faraggi, Phys. Lett. B278 (1992) 131; Nucl. Phys. B403 (1992) 101.

[18] A.E. Faraggi, Phys. Lett. B274 (1992) 47; Phys. Lett. B339 (1994) 223.

[19] A.E. Faraggi, Phys. Lett. B302 (1993) 202.

[20] A.E. Faraggi, Phys. Lett. B339 (1994) 223.

[21] A.E. Faraggi, Nucl. Phys. BB387 (1992) 239.

[22] G.B. Cleaver, A.E. Faraggi and C. Savage, Phys. Rev. D63 (2001) 066001, [hep$\mathrm{ph} / 0006331$. 
[23] G.B. Cleaver, D. Clements, A.E. Faraggi, Phys. Rev. D65 (2002) 106003, [hep$\mathrm{ph} / 0106060]$.

[24] G.B. Cleaver, A.E. Faraggi, E. Meyes, D.V. Nanopoulos, J. Perkins, and J.W. Walker, research in progress.

[25] A. Schellekens, in "Superstring Construction," (North-Holland, New York, 1989) p. 2;

A. Schellekens and N. Warner, Nucl. Phys. B313 (89) 41;

T. Kobayashi and H. Nakano, Nucl. Phys. B496 (1997) 103, [hep-th/9612066];

G.B. Cleaver and A.E. Faraggi, Int. J. Mod. Phys. A14 (1999) 2335, [hepph/9711339].

[26] M. Green and J. Schwarz, Phys. Lett. B149 (1984) 117;

M. Dine, N. Seiberg and E. Witten, Nucl. Phys. B289 (1987) 589;

J. Atick, L. Dixon and A. Sen, Nucl. Phys. B292 (1987) 109.

[27] G.B. Cleaver, J. Ellis, and D.V. Nanopoulos, Nucl. Phys. B600 (2001) 315, [hep-ph/0009338].

[28] G.B. Cleaver, J. Ellis, D.V. Nanopoulos, E. Meyes, and J. Walker, research in progress.

[29] G. B. Cleaver, A.E. Faraggi, and T. ter Veldhuis, Int. J. Mod. Phys. A16 (2001) 3565

[30] G.B. Cleaver, D.V. Nanopoulos, J. Perkins, and J.W. Walker, "Geometric Interpretation of Non-Abelian D- and F-Flat Direction Constraints," to appear. 


\section{Variations on [321] NAHE-Based Models.}

\begin{tabular}{|c|c|c|}
\hline Model Name & Gauge Group & MSSM Exotic Matter Content \\
\hline FCREU1 & $\begin{array}{l}S U(3)_{C} \times S U(2)_{L} \times U(1)_{Y} \times U(1)_{Z^{\prime}} \\
\times U(1)_{A} \times \prod_{i=1}^{5} U(1)_{i} \\
\times S U(5)_{H} \times S U(3)_{H} \\
\times U(1)_{6} \times U(1)_{7}\end{array}$ & $\begin{array}{l}\text { three }(3,1)_{-1 / 3}, \text { three }(\overline{3}, 1)_{1 / 3} \\
\text { one }(3,1)_{1 / 6}, \text { one }(\overline{3}, 1)_{-1 / 6} \\
\operatorname{six}(1,2)_{0} \\
\text { one }(1,1)_{1 / 2, Q_{A}=0} \text {, one }(1,1)_{-1 / 2, Q_{A}=0} \\
\text { three }(1,1)_{-1 / 2, Q_{A}=-1 / 2}, \text { three }(1,1)_{1 / 2, Q_{A}=1 / 2} \\
\text { three }(1,1)_{-1 / 2, Q_{A}=1 / 2} \text {, three }(1,1)_{1 / 2, Q_{A}=-1 / 2} \\
\text { three of } h_{i=1,2,3,4}, \text { three of } \bar{h}_{i=1,2,3,4}\end{array}$ \\
\hline FCREU2 & $\begin{array}{l}S U(3)_{C} \times S U(2)_{L} \times S U(2)_{C} \times U(1)_{Y^{\prime}} \\
\times U(1)_{A} \times \prod_{i=1}^{5} U(1)_{i} \\
\times S U(3)_{H} \times S U(2)_{H_{1}} \times S U(2)_{H_{2}} \\
\times \prod_{i=6}^{9} U(1)_{i}\end{array}$ & $\begin{array}{l}\text { two }(3,1)_{-1 / 3}, \text { two }(\overline{3}, 1)_{1 / 3} \\
\text { one }(3,1)_{1 / 6} \text {, one }(\overline{3}, 1)_{-1 / 6} \\
\text { three }(1,2)_{0}, \text { three }\left(1,2,2_{c}\right)_{0} \\
\text { three }(1,1)_{-1 / 2, Q_{A}=-1 / 2}, \text { three }(1,1)_{1 / 2, Q_{A}=-1 / 2} \\
\text { two of } h_{i=1,2,3} \text {, two of } \bar{h}_{i=1,2,3}\end{array}$ \\
\hline FCREU3 & $\begin{array}{l}S U(3)_{C} \times S U(2)_{L} \times U(1)_{Y} \times U(1)_{Z^{\prime}} \\
\times U(1)_{A} \times \prod_{i=1}^{5} U(1)_{i} \\
\times S U(3)_{H} \times S U(2)_{H_{1}} \times S U(2)_{H_{2}} \\
\times \prod_{i=6}^{9} U(1)_{i}\end{array}$ & $\begin{array}{l}\text { one }(3,1)_{-1 / 3}, \text { one }(\overline{3}, 1)_{1 / 3} \\
\text { one }(3,1)_{1 / 6} \text {, one }(\overline{3}, 1)_{-1 / 6} \\
\text { six }(1,2)_{0} \\
\text { one }(1,1)_{-1 / 2, Q_{A}=0} \text {, one }(1,1)_{1 / 2, Q_{A}=0} \\
\text { three }(1,1)_{-1 / 2, Q_{A}=1 / 2} \text {, three }(1,1)_{1 / 2, Q_{A}=-1 / 2} \\
\text { three }(1,1)_{-1 / 2, Q_{A}=-1 / 2}, \text { three }(1,1)_{1 / 2, Q_{A}=1 / 2} \\
\text { three of } h_{i=1,2,3,4} \text {, three of } \bar{h}_{i=1,2,3,4}\end{array}$ \\
\hline FCREU4 & $\begin{array}{l}S U(4)_{C} \times S U(2)_{L} \times U(1)_{Y^{\prime}} \\
\times U(1) \times \prod_{i=1}^{5} U(1)_{i} \\
\times S U(5)_{H} \times S U(3)_{H} \\
\times U(1)_{6} \times U(1)_{7}\end{array}$ & $\begin{array}{l}\text { no }(3,1) \text {, no }(\overline{3}, 1) \\
\text { three }(1,2)_{0} \\
\text { three }(1,2)_{1 / 2}, \text { three }(1,2)_{-1 / 2} \\
\text { one }(1,1)_{1 / 2, Q_{A}=0} \text {, one }(1,1)_{-1 / 2, Q_{A}=0} \\
\text { three }(1,1)_{1 / 2, Q_{A}=-1 / 2}, \text { three }(1,1)_{-1 / 2, Q_{A}=-1 / 2} \\
\text { two of } h_{i=1,2,3}, \text { two of } \bar{h}_{i=1,2,3}\end{array}$ \\
\hline FCREU5 & $\begin{array}{l}S U(4)_{C} \times S U(2)_{L} \times U(1)_{Y^{\prime}} \\
\times U(1)_{A} \times \prod_{i=1}^{5} U(1)_{i} \\
\times S U(3)_{H} \times S U(2)_{H_{1}} \times S U(2)_{H_{2}} \\
\times \prod_{i=6}^{9} U(1)_{i}\end{array}$ & $\begin{array}{l}\text { no }(3,1) \text {, no }(\overline{3}, 1) \\
\text { three }(1,2)_{0} \\
\text { two }(1,2)_{1 / 2}, \text { two }(1,2)_{-1 / 2} \\
\text { one }(1,1)_{1 / 2, Q_{A}=0} \text {, one }(1,1)_{-1 / 2, Q_{A}=0} \\
\text { three }(1,1)_{-1 / 2, Q_{A}=-1 / 2}, \text { three }(1,1)_{1 / 2, Q_{A}=-1 / 2} \\
\text { two of } h_{i=1,2,3}, \text { two of } \bar{h}_{i=1,2,3}\end{array}$ \\
\hline
\end{tabular}

Table A1: Gauge Groups and MSSM Exotics Fields of Models. The second column gives the gauge group for each model. The third column entry specifies the MSSMcharged exotic matter content. An exotic's representation under $S U(3)_{C} \times S U(2)_{L}$ is specified by the two numbers in brackets. Hypercharge is given by the first subscript and anomalous $U(1)$ charge is given for non-Abelian singlets by a second subscript. 


\section{GSO Phases Variations}

\begin{tabular}{|l|l|}
\hline Model Name & GSO Phase Variations \\
\hline FCREU1 & $C\left(\begin{array}{l}\mathbf{b}_{1} \\
\mathbf{b}_{1}\end{array}\right)=C\left(\begin{array}{l}\gamma \\
1\end{array}\right)=-C\left(\begin{array}{c}\gamma \\
\alpha, \beta\end{array}\right)=1$ \\
\hline FCREU2 & $C\left(\begin{array}{l}\mathbf{b}_{1} \\
\mathbf{b}_{1}\end{array}\right)=C\left(\begin{array}{l}\gamma \\
1\end{array}\right)=-C\left(\begin{array}{c}\gamma \\
\alpha, \beta\end{array}\right)=-C\left(\begin{array}{l}\beta \\
1\end{array}\right)=1$ \\
\hline FCREU3 & $C\left(\begin{array}{l}\gamma \\
1\end{array}\right)=-C\left(\begin{array}{c}\gamma \\
\alpha, \beta\end{array}\right)=-C\left(\begin{array}{l}\beta \\
1\end{array}\right)=1$ \\
\hline FCREU4 & $C\left(\begin{array}{l}\gamma \\
1\end{array}\right)=-C\left(\begin{array}{c}\gamma \\
\alpha, \beta\end{array}\right)=1$ \\
\hline FCREU5 & $C\left(\begin{array}{l}\gamma \\
1\end{array}\right)=-C\left(\begin{array}{c}\gamma \\
\alpha, \beta\end{array}\right)=-C\left(\begin{array}{l}\alpha \\
1\end{array}\right)=1$ \\
\hline
\end{tabular}

Table A2: New Models and Their GSO Phase Variations. Only the GSO phases differing from those of $[18]$ are given. 
Model FCREU1 Fields

\begin{tabular}{|c|c|c|c|c|c|c|c|c|c|c|c|c|}
\hline$F$ & $\begin{array}{l}\left(S U(3)_{C},\right. \\
\left.S U(2)_{L}\right)\end{array}$ & $Q_{Y}$ & $Q_{Z^{\prime}}$ & $Q_{A}$ & $\overline{Q_{1}}$ & $Q_{2}$ & $Q_{3}$ & $Q_{4}$ & $Q_{5}$ & $\begin{array}{l}\left(S U(5)_{H},\right. \\
\left.S U(3)_{H}\right)\end{array}$ & $Q_{6}$ & $Q_{7}$ \\
\hline$Q_{1}$ & $(3,2)$ & $1 / 6$ & $1 / 6$ & $1 / 2$ & $-1 / 2$ & $1 / 2$ & 0 & $1 / 2$ & 0 & $(1,1)$ & 0 & 0 \\
\hline$u_{1}$ & $(\overline{3}, 1)$ & $-2 / 3$ & $1 / 3$ & $1 / 2$ & $-1 / 2$ & $1 / 2$ & 0 & $-1 / 2$ & 0 & $(1,1)$ & 0 & 0 \\
\hline$d_{1}$ & $(\overline{3}, 1)$ & $1 / 3$ & $-2 / 3$ & $1 / 2$ & $-1 / 2$ & $1 / 2$ & 0 & $-1 / 2$ & 0 & $(1,1)$ & 0 & 0 \\
\hline$L_{1}$ & $(1,2)$ & $-1 / 2$ & $-1 / 2$ & $1 / 2$ & $-1 / 2$ & $1 / 2$ & 0 & $1 / 2$ & 0 & $(1,1)$ & 0 & 0 \\
\hline$e_{1}$ & $(1,1)$ & 1 & 0 & $1 / 2$ & $-1 / 2$ & $1 / 2$ & 0 & $-1 / 2$ & 0 & $(1,1)$ & 0 & 0 \\
\hline$N_{1}$ & $(1,1)$ & 0 & 1 & $1 / 2$ & $-1 / 2$ & $1 / 2$ & 0 & $-1 / 2$ & 0 & $(1,1)$ & 0 & 0 \\
\hline$Q_{2}$ & $(3,2)$ & $1 / 6$ & $1 / 6$ & $1 / 2$ & $1 / 2$ & $1 / 2$ & $-1 / 2$ & 0 & 0 & $(1,1)$ & 0 & 0 \\
\hline$u_{2}$ & $(\overline{3}, 1)$ & $-2 / 3$ & $1 / 3$ & $1 / 2$ & $1 / 2$ & $1 / 2$ & $1 / 2$ & 0 & 0 & $(1,1)$ & 0 & 0 \\
\hline$d_{2}$ & $(\overline{3}, 1)$ & $1 / 3$ & $-2 / 3$ & $1 / 2$ & $1 / 2$ & $1 / 2$ & $1 / 2$ & 0 & 0 & $(1,1)$ & 0 & 0 \\
\hline$L_{2}$ & $(1,2)$ & $-1 / 2$ & $-1 / 2$ & $1 / 2$ & $1 / 2$ & $1 / 2$ & $-1 / 2$ & 0 & 0 & $(1,1)$ & 0 & 0 \\
\hline$e_{2}$ & $(1,1)$ & 1 & 0 & $1 / 2$ & $1 / 2$ & $1 / 2$ & $1 / 2$ & 0 & 0 & $(1,1)$ & 0 & 0 \\
\hline$N_{2}$ & $(1,1)$ & 0 & 1 & $1 / 2$ & $1 / 2$ & $1 / 2$ & $1 / 2$ & 0 & 0 & $(1,1)$ & 0 & 0 \\
\hline$Q_{3}$ & $(3,2)$ & $1 / 6$ & $1 / 6$ & $1 / 2$ & 0 & -1 & 0 & 0 & $-1 / 2$ & $(1,1)$ & 0 & 0 \\
\hline$u_{3}$ & $(\overline{3}, 1)$ & $-2 / 3$ & $1 / 3$ & $1 / 2$ & 0 & -1 & 0 & 0 & $1 / 2$ & $(1,1)$ & 0 & 0 \\
\hline$d_{3}$ & $(\overline{3}, 1)$ & $1 / 3$ & $-2 / 3$ & $1 / 2$ & 0 & -1 & 0 & 0 & $1 / 2$ & $(1,1)$ & 0 & 0 \\
\hline$L_{3}$ & $(1,2)$ & $-1 / 2$ & $-1 / 2$ & $1 / 2$ & 0 & -1 & 0 & 0 & $-1 / 2$ & $(1,1)$ & 0 & 0 \\
\hline$e_{3}$ & $(1,1)$ & 1 & 0 & $1 / 2$ & 0 & -1 & 0 & 0 & $1 / 2$ & $(1,1)$ & 0 & 0 \\
\hline$N_{3}$ & $(1,1)$ & 0 & 1 & $1 / 2$ & 0 & -1 & 0 & 0 & $1 / 2$ & $(1,1)$ & 0 & 0 \\
\hline$h_{1}$ & $(1,2)$ & $-1 / 2$ & $1 / 2$ & 1 & -1 & 1 & 0 & 0 & 0 & $(1,1)$ & 0 & 0 \\
\hline$h_{2}$ & $(1,2)$ & $-1 / 2$ & $1 / 2$ & 1 & 1 & 1 & 0 & 0 & 0 & $(1,1)$ & 0 & 0 \\
\hline$h_{3}$ & $(1,2)$ & $-1 / 2$ & $1 / 2$ & 1 & 0 & -2 & 0 & 0 & 0 & $(1,1)$ & 0 & 0 \\
\hline$h_{4}$ & $(1,2)$ & $-1 / 2$ & 0 & $-1 / 4$ & $-1 / 2$ & $1 / 2$ & 0 & 0 & 0 & $(1,1)$ & 2 & 0 \\
\hline $\bar{h}_{1}$ & $(1,2)$ & $1 / 2$ & $-1 / 2$ & -1 & 1 & -1 & 0 & 0 & 0 & $(1,1)$ & 0 & 0 \\
\hline $\bar{h}_{2}$ & $(1,2)$ & $1 / 2$ & $-1 / 2$ & -1 & -1 & -1 & 0 & 0 & 0 & $(1,1)$ & 0 & 0 \\
\hline $\bar{h}_{3}$ & $(1,2)$ & $1 / 2$ & $-1 / 2$ & -1 & 0 & 2 & 0 & 0 & 0 & $(1,1)$ & 0 & 0 \\
\hline $\bar{h}_{4}$ & $(1,2)$ & $1 / 2$ & 0 & $1 / 4$ & $1 / 2$ & $-1 / 2$ & 0 & 0 & 0 & $(1,1)$ & -2 & 0 \\
\hline$D_{1}$ & $(3,1)$ & $-1 / 3$ & $-1 / 3$ & 1 & 0 & 1 & 0 & 0 & 0 & $(1,1)$ & 0 & 0 \\
\hline$D_{2}$ & $(3,1)$ & $-1 / 3$ & $-1 / 3$ & -1 & 0 & -1 & 0 & 0 & 0 & $(1,1)$ & 0 & 0 \\
\hline$D_{3}$ & $(3,1)$ & $-1 / 3$ & $1 / 6$ & $1 / 4$ & $-1 / 2$ & $-1 / 2$ & 0 & 0 & 0 & $(1,1)$ & -2 & 0 \\
\hline$D_{4}$ & $(3,1)$ & $1 / 6$ & $1 / 6$ & 0 & 0 & 0 & $1 / 2$ & $1 / 2$ & $1 / 2$ & $(1,1)$ & $1 / 2$ & $-15 / 2$ \\
\hline $\bar{D}_{1}$ & $(\overline{3}, 1)$ & $1 / 3$ & $1 / 3$ & -1 & 0 & -1 & 0 & 0 & 0 & $(1,1)$ & 0 & 0 \\
\hline $\bar{D}_{2}$ & $(\overline{3}, 1)$ & $1 / 3$ & $1 / 3$ & 1 & 0 & 1 & 0 & 0 & 0 & $(1,1)$ & 0 & 0 \\
\hline $\bar{D}_{3}$ & $(\overline{3}, 1)$ & $1 / 3$ & $1 / 6$ & $-1 / 4$ & $1 / 2$ & $1 / 2$ & 0 & 0 & 0 & $(1,1)$ & 2 & 0 \\
\hline $\bar{D}_{4}$ & $(\overline{3}, 1)$ & $-1 / 6$ & $-1 / 6$ & 0 & 0 & 0 & $-1 / 2$ & $-1 / 2$ & $-1 / 2$ & $(1,1)$ & $-1 / 2$ & $15 / 2$ \\
\hline$X_{1}$ & $(1,2)$ & 0 & 0 & $1 / 2$ & $-1 / 2$ & $1 / 2$ & $1 / 2$ & 0 & $1 / 2$ & $(1,1)$ & $-1 / 2$ & $15 / 2$ \\
\hline$X_{2}$ & $(1,2)$ & 0 & 0 & $1 / 2$ & $1 / 2$ & $1 / 2$ & 0 & $-1 / 2$ & $1 / 2$ & $(1,1)$ & $-1 / 2$ & $15 / 2$ \\
\hline$X_{3}$ & $(1,2)$ & 0 & 0 & $1 / 2$ & 0 & -1 & $1 / 2$ & $-1 / 2$ & 0 & $(1,1)$ & $-1 / 2$ & $15 / 2$ \\
\hline $\bar{X}_{1}$ & $(1,2)$ & 0 & 0 & $-1 / 2$ & $1 / 2$ & $-1 / 2$ & $1 / 2$ & 0 & $1 / 2$ & $(1,1)$ & $1 / 2$ & $-15 / 2$ \\
\hline $\bar{X}_{2}$ & $(1,2)$ & 0 & 0 & $-1 / 2$ & $-1 / 2$ & $-1 / 2$ & 0 & $-1 / 2$ & $1 / 2$ & $(1,1)$ & $1 / 2$ & $-15 / 2$ \\
\hline $\bar{X}_{3}$ & $(1,2)$ & 0 & 0 & $-1 / 2$ & 0 & 1 & $1 / 2$ & $-1 / 2$ & 0 & $(1,1)$ & $1 / 2$ & $-15 / 2$ \\
\hline
\end{tabular}

Table A3: Model FCREU1 Fields and Their Charges. 


\begin{tabular}{|c|c|c|c|c|c|c|c|c|c|c|c|c|}
\hline$F$ & $\begin{array}{l}\left(S U(3)_{C},\right. \\
\left.S U(2)_{L}\right)\end{array}$ & $Q_{Y}$ & $Q_{Z^{\prime}}$ & $Q_{A}$ & $Q_{1}$ & $Q_{2}$ & $Q_{3}$ & $Q_{4}$ & $Q_{5}$ & $\begin{array}{l}\left(S U(5)_{H},\right. \\
\left.S U(3)_{H}\right)\end{array}$ & $Q_{6}$ & $Q_{7}$ \\
\hline$A_{1}$ & $(1,1)$ & $1 / 2$ & $1 / 2$ & 0 & $\overline{0}$ & $\overline{0}$ & $1 / 2$ & $1 / 2$ & $-1 / 2$ & $(1,1)$ & $-1 / 2$ & $15 / 2$ \\
\hline$A_{2}$ & $(1,1)$ & $-1 / 2$ & $1 / 2$ & $-1 / 2$ & $-1 / 2$ & $-1 / 2$ & 0 & $1 / 2$ & $-1 / 2$ & $(1,1)$ & $-1 / 2$ & $15 / 2$ \\
\hline$A_{3}$ & $(1,1)$ & $-1 / 2$ & $1 / 2$ & $-1 / 2$ & 0 & 1 & $-1 / 2$ & $1 / 2$ & 0 & $(1,1)$ & $-1 / 2$ & $15 / 2$ \\
\hline$A_{4}$ & $(1,1)$ & $-1 / 2$ & $1 / 2$ & $-1 / 2$ & $1 / 2$ & $-1 / 2$ & $-1 / 2$ & 0 & $-1 / 2$ & $(1,1)$ & $-1 / 2$ & $15 / 2$ \\
\hline$A_{5}$ & $(1,1)$ & $1 / 2$ & $-1 / 2$ & $-1 / 2$ & $-1 / 2$ & $-1 / 2$ & 0 & $1 / 2$ & $-1 / 2$ & $(1,1)$ & $-1 / 2$ & $15 / 2$ \\
\hline$A_{6}$ & $(1,1)$ & $1 / 2$ & $-1 / 2$ & $-1 / 2$ & 0 & 1 & $-1 / 2$ & $1 / 2$ & 0 & $(1,1)$ & $-1 / 2$ & $15 / 2$ \\
\hline$A_{7}$ & $(1,1)$ & $1 / 2$ & $-1 / 2$ & $-1 / 2$ & $1 / 2$ & $-1 / 2$ & $-1 / 2$ & 0 & $-1 / 2$ & $(1,1)$ & $-1 / 2$ & $15 / 2$ \\
\hline $\bar{A}_{1}$ & $(1,1)$ & $-1 / 2$ & $-1 / 2$ & 0 & 0 & 0 & $-1 / 2$ & $-1 / 2$ & $1 / 2$ & $(1,1)$ & $1 / 2$ & $-15 / 2$ \\
\hline $\bar{A}_{2}$ & $(1,1)$ & $1 / 2$ & $-1 / 2$ & $1 / 2$ & $1 / 2$ & $1 / 2$ & 0 & $1 / 2$ & $-1 / 2$ & $(1,1)$ & $1 / 2$ & $-15 / 2$ \\
\hline $\bar{A}_{3}$ & $(1,1)$ & $1 / 2$ & $-1 / 2$ & $1 / 2$ & 0 & -1 & $-1 / 2$ & $1 / 2$ & 0 & $(1,1)$ & $1 / 2$ & $-15 / 2$ \\
\hline $\bar{A}_{4}$ & $(1,1)$ & $1 / 2$ & $-1 / 2$ & $1 / 2$ & $-1 / 2$ & $1 / 2$ & $-1 / 2$ & 0 & $-1 / 2$ & $(1,1)$ & $1 / 2$ & $-15 / 2$ \\
\hline $\bar{A}_{5}$ & $(1,1)$ & $-1 / 2$ & $1 / 2$ & $1 / 2$ & $1 / 2$ & $1 / 2$ & 0 & $1 / 2$ & $-1 / 2$ & $(1,1)$ & $1 / 2$ & $-15 / 2$ \\
\hline $\bar{A}_{6}$ & $(1,1)$ & $-1 / 2$ & $1 / 2$ & $1 / 2$ & 0 & -1 & $-1 / 2$ & $1 / 2$ & 0 & $(1,1)$ & $1 / 2$ & $-15 / 2$ \\
\hline $\bar{A}_{7}$ & $(1,1)$ & $-1 / 2$ & $1 / 2$ & $1 / 2$ & $-1 / 2$ & $1 / 2$ & $-1 / 2$ & 0 & $-1 / 2$ & $(1,1)$ & $1 / 2$ & $-15 / 2$ \\
\hline$\Phi_{1}$ & $(1,1)$ & 0 & 0 & 0 & 0 & 0 & 0 & 0 & 0 & $(1,1)$ & 0 & 0 \\
\hline$\Phi_{2}$ & $(1,1)$ & 0 & 0 & 0 & 0 & 0 & 0 & 0 & 0 & $(1,1)$ & 0 & 0 \\
\hline$\Phi_{3}$ & $(1,1)$ & 0 & 0 & 0 & 0 & 0 & 0 & 0 & 0 & $(1,1)$ & 0 & 0 \\
\hline$\Phi_{12}$ & $(1,1)$ & 0 & 0 & 0 & -2 & 0 & 0 & 0 & 0 & $(1,1)$ & 0 & 0 \\
\hline$\Phi_{23}$ & $(1,1)$ & 0 & 0 & 0 & 1 & -3 & 0 & 0 & 0 & $(1,1)$ & 0 & 0 \\
\hline$\Phi_{31}$ & $(1,1)$ & 0 & 0 & 0 & -1 & -3 & 0 & 0 & 0 & $(1,1)$ & 0 & 0 \\
\hline $\bar{\Phi}_{3}$ & $(1,1)$ & 0 & 0 & 0 & 2 & 0 & 0 & 0 & 0 & $(1,1)$ & 0 & 0 \\
\hline $\bar{\Phi}_{23}$ & $(1,1)$ & 0 & 0 & 0 & -1 & 3 & 0 & 0 & 0 & $(1,1)$ & 0 & 0 \\
\hline $\bar{\Phi}_{31}$ & $(1,1)$ & 0 & 0 & 0 & 1 & 3 & 0 & 0 & 0 & $(1,1)$ & 0 & 0 \\
\hline$S_{1}$ & $(1,1)$ & 0 & 0 & 0 & -1 & 0 & -1 & 0 & 0 & $(1,1)$ & 0 & 0 \\
\hline$S_{2}$ & $(1,1)$ & 0 & 0 & 0 & -1 & 0 & 1 & 0 & 0 & $(1,1)$ & 0 & 0 \\
\hline$S_{3}$ & $(1,1)$ & 0 & 0 & 0 & -1 & 0 & 0 & -1 & 0 & $(1,1)$ & 0 & 0 \\
\hline$S_{4}$ & $(1,1)$ & 0 & 0 & 0 & -1 & 0 & 0 & 1 & 0 & $(1,1)$ & 0 & 0 \\
\hline$S_{5}$ & $(1,1)$ & 0 & 0 & 0 & -1 & 0 & 0 & 0 & -1 & $(1,1)$ & 0 & 0 \\
\hline$S_{6}$ & $(1,1)$ & 0 & 0 & 0 & -1 & 0 & 0 & 0 & 1 & $(1,1)$ & 0 & 0 \\
\hline$S_{7}$ & $(1,1)$ & 0 & $1 / 2$ & $3 / 4$ & $-1 / 2$ & $-3 / 2$ & 0 & 0 & 0 & $(1,1)$ & 2 & 0 \\
\hline$S_{8}$ & $(1,1)$ & 0 & $1 / 2$ & $3 / 4$ & $1 / 2$ & $3 / 2$ & 0 & 0 & 0 & $(1,1)$ & 2 & 0 \\
\hline$S_{9}$ & $(1,1)$ & 0 & $1 / 2$ & $-5 / 4$ & $1 / 2$ & $-1 / 2$ & 0 & 0 & 0 & $(1,1)$ & 2 & 0 \\
\hline $\bar{S}_{1}$ & $(1,1)$ & 0 & 0 & 0 & 1 & 0 & 1 & 0 & 0 & $(1,1)$ & 0 & 0 \\
\hline $\bar{S}_{2}$ & $(1,1)$ & 0 & 0 & 0 & 1 & 0 & -1 & 0 & 0 & $(1,1)$ & 0 & 0 \\
\hline $\bar{S}_{3}$ & $(1,1)$ & 0 & 0 & 0 & 1 & 0 & 0 & 1 & 0 & $(1,1)$ & 0 & 0 \\
\hline $\bar{S}_{4}$ & $(1,1)$ & 0 & 0 & 0 & 1 & 0 & 0 & -1 & 0 & $(1,1)$ & 0 & 0 \\
\hline $\bar{S}_{5}$ & $(1,1)$ & 0 & 0 & 0 & 1 & 0 & 0 & 0 & 1 & $(1,1)$ & 0 & 0 \\
\hline $\bar{S}_{6}$ & $(1,1)$ & 0 & 0 & 0 & 1 & 0 & 0 & 0 & -1 & $(1,1)$ & 0 & 0 \\
\hline $\bar{S}_{7}$ & $(1,1)$ & 0 & $-1 / 2$ & $-3 / 4$ & $1 / 2$ & $3 / 2$ & 0 & 0 & 0 & $(1,1)$ & -2 & 0 \\
\hline $\bar{S}_{8}$ & $(1,1)$ & 0 & $-1 / 2$ & $-3 / 4$ & $-1 / 2$ & $-3 / 2$ & 0 & 0 & 0 & $(1,1)$ & -2 & 0 \\
\hline $\bar{S}_{9}$ & $(1,1)$ & 0 & $-1 / 2$ & $5 / 4$ & $-1 / 2$ & $1 / 2$ & 0 & 0 & 0 & $(1,1)$ & -2 & 0 \\
\hline
\end{tabular}

Table A3 continued: Model FCREU1 Fields and Their Charges. 


\begin{tabular}{|l||c|cccccccc|c|cc|}
\hline$F$ & $\begin{array}{c}S U(3)_{C}, \\
\left.S U(2)_{L}\right)\end{array}$ & $Q_{Y}$ & $Q_{Z^{\prime}}$ & $Q_{A}$ & $Q_{1}$ & $Q_{2}$ & $Q_{3}$ & $Q_{4}$ & $Q_{5}$ & $\begin{array}{c}\left(S U(5)_{H},\right. \\
\left.S U(3)_{H}\right)\end{array}$ & $Q_{6}$ & $Q_{7}$ \\
\hline$F_{1}$ & $(1,1)$ & 0 & $-1 / 2$ & $-1 / 4$ & $-1 / 2$ & $1 / 2$ & 0 & 0 & 0 & $(5,1)$ & -1 & -3 \\
$F_{2}$ & $(1,1)$ & 0 & 0 & 1 & 0 & 1 & 0 & 0 & $1 / 2$ & $(5,1)$ & 1 & -3 \\
$F_{3}$ & $(1,1)$ & 0 & 0 & 1 & $-1 / 2$ & $-1 / 2$ & $1 / 2$ & 0 & 0 & $(5,1)$ & 1 & -3 \\
$F_{4}$ & $(1,1)$ & 0 & 0 & 1 & $1 / 2$ & $-1 / 2$ & 0 & $-1 / 2$ & 0 & $(5,1)$ & 1 & -3 \\
$\bar{F}_{1}$ & $(1,1)$ & 0 & $1 / 2$ & $1 / 4$ & $1 / 2$ & $-1 / 2$ & 0 & 0 & 0 & $(\overline{5}, 1)$ & 1 & 3 \\
$\bar{F}_{2}$ & $(1,1)$ & 0 & 0 & 1 & 0 & 1 & 0 & 0 & $-1 / 2$ & $(\overline{5}, 1)$ & -1 & 3 \\
$\bar{F}_{3}$ & $(1,1)$ & 0 & 0 & 1 & $-1 / 2$ & $-1 / 2$ & $-1 / 2$ & 0 & 0 & $(\overline{5}, 1)$ & -1 & 3 \\
$\bar{F}_{4}$ & $(1,1)$ & 0 & 0 & 1 & $1 / 2$ & $-1 / 2$ & 0 & $1 / 2$ & 0 & $(\overline{5}, 1)$ & -1 & 3 \\
\hline$K_{1}$ & $(1,1)$ & 0 & $1 / 2$ & $1 / 4$ & $-1 / 2$ & $-1 / 2$ & 0 & 0 & 0 & $(1,3)$ & 1 & -5 \\
$K_{2}$ & $(1,1)$ & 0 & 0 & 1 & $1 / 2$ & $-1 / 2$ & 0 & $-1 / 2$ & 0 & $(1,3)$ & -1 & -5 \\
$K_{3}$ & $(1,1)$ & 0 & 0 & 1 & $-1 / 2$ & $-1 / 2$ & $1 / 2$ & 0 & 0 & $(1,3)$ & -1 & -5 \\
$K_{4}$ & $(1,1)$ & 0 & 0 & 1 & 0 & 1 & 0 & 0 & $1 / 2$ & $(1,3)$ & -1 & -5 \\
$\bar{K}_{1}$ & $(1,1)$ & 0 & $-1 / 2$ & $-1 / 4$ & $1 / 2$ & $-1 / 2$ & 0 & 0 & 0 & $(1, \overline{3})$ & -1 & 5 \\
$\bar{K}_{2}$ & $(1,1)$ & 0 & 0 & 1 & $1 / 2$ & $-1 / 2$ & 0 & $1 / 2$ & 0 & $(1, \overline{3})$ & 1 & 5 \\
$\bar{K}_{3}$ & $(1,1)$ & 0 & 0 & 1 & $-1 / 2$ & $-1 / 2$ & $-1 / 2$ & 0 & 0 & $(1, \overline{3})$ & 1 & 5 \\
$\bar{K}_{4}$ & $(1,1)$ & 0 & 0 & 1 & 0 & 1 & 0 & 0 & $-1 / 2$ & $(1, \overline{3})$ & 1 & 5 \\
\hline
\end{tabular}

Table A3 continued: Model FCREU1 Fields and Their Charges. 